\title{
Design of microcavity resonators for single-atom detection
}

\author{
Michael Rosenblit, ${ }^{a}$ Peter Horak, ${ }^{b}$ Eyal Fleminger, ${ }^{a}$ Yonathan \\ Japha, $^{a}$ and Ron Folman ${ }^{a}$ \\ ${ }^{a}$ Department of Physics and The Weiss Family Laboratory for Nano-scale System, \\ Ben Gurion University of the Negev, Be'er Sheva 84105, Israel. \\ ${ }^{b}$ Research Centre, University of Southampton, \\ Southampton SO17 1BJ, United Kingdom.
}

\begin{abstract}
Whispering gallery modes of a microdisk resonator are useful for the optical detection of single rubidium and cesium atoms near the surface of a substrate. Light is coupled into two high-Q whispering-gallery modes of the disk which can provide attractive and/or repulsive potentials, respectively, via their evanescent fields. The sum potential, including van der Waals/Casimir-Polder surface forces, may be tuned to exhibit a minimum at distances on the order of 100 $\mathrm{nm}$ from the disk surface. Simultaneously optically trapping and detecting is possible, with the back-action of an atom held in this trap on the light fields being sufficiently strong to provide a measurable effect. Atom trapping and detection depend on a variety of system parameters and experimental realizations differ for different atoms.
\end{abstract}

Keywords: atom detection, atom trapping, micro resonators, optics, photonics.

\section{INTRODUCTION}

The possibility of storing light in optical resonators has long been at the core of a wide range of applications. Modern technology has allowed for a reduction of resonator size down to the regime of micrometers, and thus is poised to reach the ultimate limit of devices comparable in size to a few wavelengths of light, while still maintaining good optical quality. Optical micro-resonators are today routinely applied in fields as varied as optical telecommunications [1, 2], and biological or chemical sensors [3].

Several different realizations of micro-resonators are currently under investigation in the context of their integration on chips for atom optics, so-called atom chips [4, 5]. Examples include Fabry-Perot fiber cavities [6], photonic bandgap structures [7], and microdisk resonators $[8,9]$. The latter possibility is attractive, since it combines the high optical quality of the much-studied microsphere [10-13] with advanced micro-fabrication and integration technology.

With a recently developed fabrication technique, the finesse of integrable microdisk resonators has been increased by several orders of magnitude [14-16]. This takes the quality of such devices to a level where effects of quantum electrodynamics can readily be observed and exploited. Integrated into atom chips, this may have a significant impact in contexts such as cavity QED [17-20], single photon sources [21-23], manipulation of matter waves in interferometric sensors [24-28], atomic clocks [29], and the quantum computer [30, 31].

In Ref. 8, we suggested the use of whispering gallery modes (WGMs) of a toroidal microcavity for single-atom detection on an atom chip. However, the initial calculations neglected atomic motion by assuming the atom to be static at a fixed distance from the microdisk surface. More recently, we investigated the possibility of atom trapping by the same optical modes which are used for atom detection [9]. We showed that sufficiently deep potential minima can in fact be created by a careful choice of parameters to hold the atom in place during the detection process. In this paper we investigate single-atom detection for rubidium and cesium atoms, near the surface of a microdisk including, van der Waals and Casimir surface forces, and compare our theory with recent experiments $[32,33]$.

\section{SYSTEM DESIGN}

A schematic of the system under investigation is shown in Figure 1. The optical part consists of a toroidal micro-cavity resonator which is coupled to a tapered optical waveguide or fiber for input and output coupling. The disk is assumed to support high-finesse WGM 


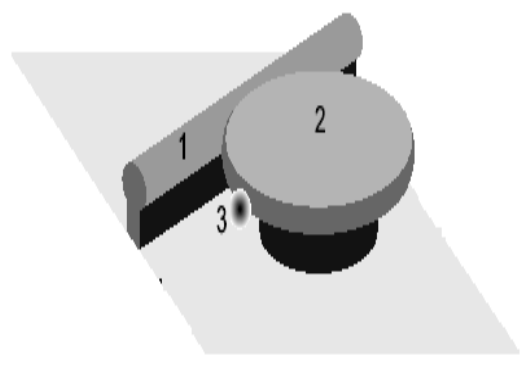

Fig. 1. The structure under consideration: (1) linear waveguide coupler, (2) microdisk, (3) atomic cloud.

near the wavelengths of the D1 and D2 lines of the alkali atoms used in experiments, i.e., around $795 \mathrm{~nm}$ and $780 \mathrm{~nm}$ for rubidium, and around $895 \mathrm{~nm}$ and $852 \mathrm{~nm}$ for cesium. The atoms are initially loaded into a magnetic microtrap with the resonator fields switched off. By switching the fields on, the atoms are then transferred into a much stronger optical trap formed by two modes of opposite detuning and the trapped atoms are finally optically detected. Another possibility is to detect atoms during free-fall using only one blue-de tuned mode to compensate for the attractive van der Waals or Casimir-Polder forces. The main optical properties of a selection of microdisk modes suitable for these schemes are summarized in Table 1.

Table 1. Optical properties of selected WGMs. $D$ is the disk diameter, $l$ is the longitudinal mode index (the radial index is $q=1), Q$ is the quality factor for a waveguide-disk gap of $0.5 \mu \mathrm{m}$, and $g_{0}$ is the single-photon Rabi frequency for an atom at the disk boundary.

\begin{tabular}{cccccc}
\hline Atom & $D(\mu \mathrm{m})$ & $l$ & $\lambda(\mathrm{nm})$ & $Q / 10^{6}$ & $g_{0}(\mathrm{MHz})$ \\
\hline $\mathrm{Rb}$ & 30 & 167 & 778.73 & 3.0 & 102.6 \\
& 30 & 163 & 797.2 & 2.27 & 105.0 \\
& 45 & 254 & 777.2 & 10 & 68.3 \\
& 45 & 253 & 780.15 & 12 & 68.5 \\
\hline Cs & 45 & 231 & 851.9 & 1.99 & 74.9 \\
& 45 & 232 & 848.3 & 2.07 & 74.6 \\
& 44 & 226 & 850.9 & 1.95 & 76.5 \\
& 44 & 214 & 897.3 & 1.12 & 80.7 \\
\hline
\end{tabular}

\section{FORCES ON ATOMS NEAR A MICRODISK}

In this section we investigate in detail the forces on an atom situated near the surface of a microdisk: forces generated by the magnetic microtrap, van der Waals and Casimir-Polder forces due to the interaction with the dielectric surface, and optical forces induced by the evanescent fields of the light confined in the resonator modes. Here we make the simplifying assumption that these forces act on the atoms individually, that is, the total potential is the simple sum of the magnetic, atom-surface, and light contributions:

$$
V=V_{\text {mag }}+V_{\mathrm{AS}}+V_{\text {light }} .
$$

In the following section we discuss these potentials individually. 


\subsection{Surface potential}

Close to the dielectric surface of the microdisk, an atom experiences an effective potential due to the mutual polarizability of the atom and the microdisk dielectric material. Two different forms of this potential have been described in the literature. At very short distances this potential is a van der Waals potential scaling as $1 / r^{3}$, where $r$ is the distance from the surface, while at larger distances on the order of one wavelength of the atomic transition, a Casimir-Polder potential is found which scales as $1 / r^{4}$ [34]. For a ground-state atom these atom-surface potentials are usually attractive.

The van der Waals potential for an isotropic atom near a dielectric material with refractive index $n$ takes the asymptotic form

$$
V_{v d W}(x)=-\left(\frac{n^{2}-1}{n^{2}+1}\right) \frac{e^{2} R^{2}}{6 \pi \epsilon_{0}(2 r)^{3}}
$$

where $e$ is the electron charge and $R^{2}$ is the expectation value of the square of the atomic radius. The Casimir-Polder potential for an isotropic atom is given by

$$
V_{C P}(x)=-\frac{\alpha_{0} \hbar c}{2 \pi^{2} \epsilon_{0}(2 r)^{4}}\left(2 c_{4}^{\|}+c_{4}^{\perp}\right)
$$

where the coefficients $c_{4}^{\|}$and $c_{4}^{\perp}$ are functions of the refractive index $n$ (see Ref. 34). The $c_{4}$ coefficients range from 0 for $n=1$ to 1 for $n \rightarrow \infty$ (a perfect conductor). In the intermediate regime of distances (on the order of $100 \mathrm{~nm}$ ), the exact atom-surface potential can be calculated numerically. However, for simplicity we approximate the potential $V_{\mathrm{AS}}$ at each point by the maximum of the expressions given in Eqs. (2) and (3).

\subsection{Optical potential}

We consider atoms close to the surface of the microdisk, coupled to the evanescent fields of two WGMs as specified in Table 1. One of the two modes is blue-detuned with respect to the atomic D2 line and thus forms a repulsive potential, whereas the second mode is red-detuned with respect to the D1 line, which provides an attractive potential. Because of the shorter decay length of the blue-detuned mode, the two field intensities can be adjusted to provide a potential minimum at a certain distance from the microdisk surface [35-41].

The optical potential is given by the sum of the two individual potentials as

$$
V_{\text {light }}(\mathbf{x}) \approx V_{\text {light }, b}(\mathbf{x})+V_{\text {light }, r}(\mathbf{x}) \approx \frac{\hbar\left|\Omega_{b}(\mathbf{x})\right|^{2}}{4 \Delta_{b}}+\frac{\hbar\left|\Omega_{r}(\mathbf{x})\right|^{2}}{4 \Delta_{r}}
$$

Here $\Delta_{b}\left(\Delta_{r}\right)$ is the detuning of the blue (red) mode from the corresponding transition and $\Omega_{i}(\mathbf{x})=2 g_{i}(\mathbf{x}) \sqrt{N_{i}}$, where $g_{i}(\mathbf{x})$ is the single-photon Rabi frequency of mode $i$ at the position of the atom and $N_{i}$ is the photon number. Examples of the total optical potentials are shown in Fig. 2. Note that there is a subtle balance between the strong potentials of the individual light fields which yields an overall potential depth on the order of $100 \mu \mathrm{K}$. The effect of the atom-surface potential, as discussed in Sec. 3.1, is to provide an additional attractive potential for the atom very close to the surface. This reduces the depth of the total potential $V$, and shifts the position of the trap minimum closer to the surface.

In the vicinity of the microdisk, the optical potentials can be approximated by decaying exponentials, $V_{i}(r)=V_{i 0} e^{-\alpha_{i} r}$ for modes $i=r, b$. The potential minimum is

$$
V\left(r_{\min }\right)=-V_{b 0} e^{-\alpha r_{\min }} \frac{\alpha_{b}-\alpha_{r}}{\alpha_{r}}
$$

and is found at a distance

$$
r_{\text {min }}=\frac{1}{\alpha_{b}-\alpha_{r}} \log \frac{\left|V_{b 0}\right| \alpha_{b}}{\left|V_{r 0}\right| \alpha_{r}} .
$$



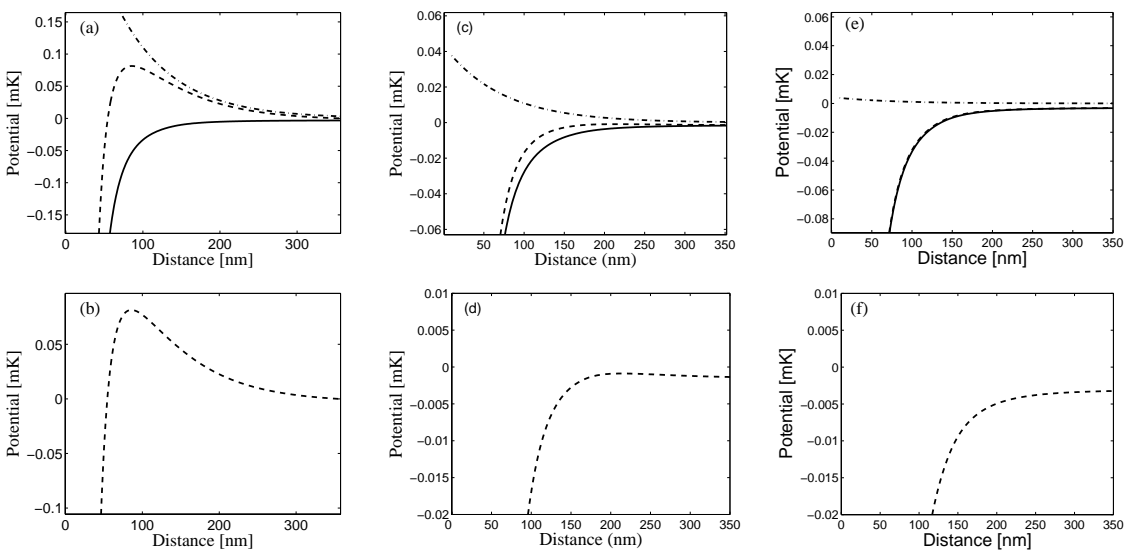

Fig. 2. Potentials formed for Cs atoms by blue-detuned light (dash-dotted line), surface interactions (solid), and the sum of these potentials (dashed). Figures in the upper row are for input light intensities of $20 \mathrm{nW}, 2 \mathrm{nW}$ and $200 \mathrm{pW}$, respectively. The lower row of figures show only the sum potentials, with an exaggerated vertical scale. For the lowest intensity there is a simple attraction to the disk edge in the evanescent field range. For the highest intensity a barrier is formed because of the blue-detuned repulsion.

The harmonic oscillator frequency in the radial direction is therefore given by

$$
\omega_{r}=\sqrt{\alpha_{b} \alpha_{r} V\left(r_{\min }\right) / m},
$$

which is typically on the order of $100 \mathrm{kHz}$.

The light fields can also be used to create a true three-dimensional $(3 \mathrm{D})$ trap. In the vertical direction, the finite size of the disk leads to potential gradients in this direction. The field amplitudes are approximately proportional to $\cos (\pi z / H)$, where $H$ is the height of the disk and $z=0$ coincides with the disk center. Likewise, trapping in the azimuthal direction can be achieved by coupling light simultaneously from both sides into the reddetuned microdisk mode to create a standing wave pattern of the WGM. The total 3D trapping potential is then given by

$$
V(x, y, z)=V_{b 0} e^{-\alpha_{b} x} \cos ^{2}(\pi z / H)+V_{r 0} e^{-\alpha_{r} x} \cos ^{2}(\pi z / H) \cos ^{2}\left(2 l_{r} y / D\right),
$$

where $l_{r}$ is the windingnumber of the red-detuned WGM. The harmonic oscillator frequencies in the $y$ and $z$ directions are then given by

$$
\omega_{y}=\frac{2 l_{r}}{D} \sqrt{\left|V_{r 0}\right| / m}, \quad \omega_{z}=\frac{\pi}{H} \sqrt{\left|V\left(r_{\min }\right)\right| / m} .
$$

These frequencies are typically a few $\mathrm{kHz}$, i.e., an order of magnitude smaller than the radial trapping frequency.

\subsection{Magnetic trap potential}

The atoms are initially prepared in a magnetic microtrap on the atom chip in the absence of light fields. In a simple case, the magnetic potential is created by the interaction of the magnetic field of a current-carrying wire, a homogeneous field $B_{0}$ orthogonal to the wire, and a homogeneous field $B_{\text {offset }}$ parallel to the wire. This forms a minimum of the total magnetic field above the wire which acts as an attractive potential for atoms in the weak-field seeking Zeeman sublevels. However, as was shown in Ref. 9, magnetic trapping in the presence of the evanescent light fields from the disk would be difficult to achieve in practice, even in the direction along the perimeter of the disk where no optical dipole forces exist. Therefore, we do not include magnetic potentials here at all, and consider only optical trapping potentials. 


\section{ATOM DETECTION}

The principles of the detection scheme have already been presented elsewhere [8, 9]. In brief, laser light (resonant with the two modes under consideration) is launched into the linear waveguide, from which it is coupled into the microdisk. The interaction with the atom changes the phase of the light fields, which can be measured at the output of the linear waveguide by a balanced homodyne detector.

We consider two coherent modes with complex amplitudes $\alpha_{+}$and $\alpha_{-}$traveling in opposing directions, and assume atom-light coupling to these modes via the respective singlephoton Rabi frequencies $g_{+}$and $g_{-}$. The coupling of these two modes due to imperfections in the disk is accounted for by the introduction of the complex coefficient $\epsilon$, and the model includes the possibility of pumping from either direction with rates $\eta_{+}$and $\eta_{-}[8]$.

After ignoring the external motion of the atom, the Hamiltonian of this system can be written as $(\hbar=1)$

$$
\begin{aligned}
H= & -\Delta_{a} \sigma_{11}-\Delta_{c}\left(a_{+}^{\dagger} a_{-}+a_{-}^{\dagger} a_{-}\right)-i\left(g_{+} a_{+}^{\dagger} \sigma_{01}-g_{+}^{*} \sigma_{10} a_{+}\right)-i \eta_{+}\left(a_{+}-a_{+}^{\dagger}\right) \\
& -i\left(g_{-} a_{-}^{\dagger} \sigma_{01}-g_{-}^{*} \sigma_{10} a_{-}\right)-i \eta_{-}\left(a_{-}-a_{-}^{\dagger}\right)-i\left(\epsilon a_{+}^{\dagger} a_{-}-\epsilon^{*} a_{-}^{\dagger} a_{+}\right),
\end{aligned}
$$

where $\Delta_{a}$ and $\Delta_{c}$ are the atomic and resonator detunings, respectively; $\sigma_{10}$ and $\sigma_{01}$ are the atomic raising and lowering operators; and $a_{ \pm}^{\dagger}$ and $a_{ \pm}$are the mode creation and annihilation operators. Here the energy of the lower atomic state has been set to zero. The equation of motion for the density operator of the system can be written as

$$
\frac{d}{d t} \rho=-i[H, \rho]+L \rho
$$

where $L$ is the usual linear operator describing cavity and atomic decay with rates $\kappa$ and $\Gamma$, respectively.

Assuming a factorized density operator $\rho$, we find that the equations of motion for the elements of the atomic density operator as follows:

$$
\begin{aligned}
& \frac{d}{d t} \rho_{10}=\left(-\Gamma+i \Delta_{a}\right) \rho_{10}+\left(g_{+}^{*} \alpha_{+}+g_{-}^{*} \alpha_{-}\right)\left(\rho_{00}-\rho_{11}\right) \\
& \frac{d}{d t} \rho_{11}=-2 \Gamma \rho_{11}+\left(g_{+}^{*} \alpha_{+}+g_{-}^{*} \alpha_{-}\right) \rho_{01}+\left(g_{+} \alpha_{+}^{*}+g_{-} \alpha_{-}^{*}\right) \rho_{10} .
\end{aligned}
$$

The coherent state amplitudes $\alpha_{ \pm}$obey the equations of motion

$$
\frac{d}{d t} \alpha_{+}=\left(i \Delta_{c}-\kappa\right) \alpha_{+}-g_{+} \rho_{10}+\eta_{+}-\epsilon \alpha_{-}, \quad \frac{d}{d t} \alpha_{-}=\left(i \Delta_{c}-\kappa\right) \alpha_{-}-g_{-} \rho_{10}+\eta_{-}+\epsilon^{*} \alpha_{+} .
$$

In this work we are only interested in the stationary solution of these equations of motion. To this end, we first solve the linear set of equations (13) with respect to $\alpha_{ \pm}$. The resulting expressions for $\alpha_{ \pm}$are linear in $\rho_{10}$ and can be inserted into Eq. (11). From this and using $\rho_{11}+\rho_{00}=1$, we obtain $\rho_{10}$ as a function of $\rho_{11}$. This expression for $\rho_{10}$ and the corresponding results for $\alpha_{ \pm}$can be inserted into Eq. (12) to give a real-valued nonlinear equation in $\rho_{11}$ which can be solved by standard numerical techniques. The output field of the linear waveguide is the superposition of the waveguide input field transmitted through the waveguide-disk coupler and the light coupled out of the disk [8].

The Rabi frequency $g_{ \pm}$is given by

$$
g_{ \pm}=E_{W G M}\left(\mathbf{x}_{a}\right)\left[\frac{3 \Gamma c^{3}}{\omega^{2} d_{y} \int r n(r)^{2}\left|E_{W G M}(r)\right|^{2} d r}\right]^{1 / 2}
$$

where $n(r)$ describes the refractive index profile inside and outside the microdisk, $d_{y}$ is the disk height, $\omega$ is the atomic transition frequency, and $\mathbf{x}_{a}=\left(r_{a}, \phi_{a}\right)$ is the atomic position in the evanescent field of the disk modes. The $g_{ \pm}$dependence on the distance from the disk 


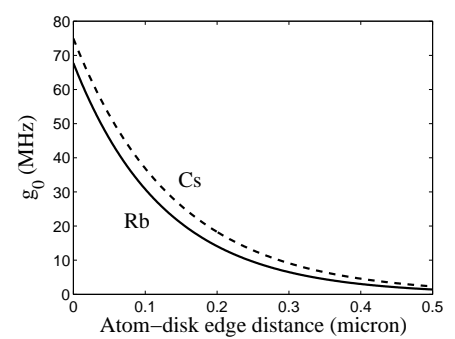

Fig. 3. Rabi frequency divided on $2 \pi$ vs. atom-disk distance. Solid curve: Rb atom, $D=45 \mu \mathrm{m}$, mode indices $(l, q)=(254,1)$; dashed: Cs atom, $D=45 \mu \mathrm{m},(l, q)=(231,1)$.

is given in Fig. 3, and the maximum values for $g_{ \pm}$at the disk surface for several selected modes are given in Table .

The signal-to-noise ratio of this atom-detection scheme is given by

$$
S=4 \sqrt{\tau}\left|A_{i n}\right| \frac{\kappa_{T} g^{2}}{\Delta \kappa^{2}}
$$

where $\tau$ is the measurement time and $A_{\text {in }}$ is the amplitude of the pump light in the linear waveguide, normalized such that $\left|A_{i n}\right|^{2}$ is the power in units of photons per second. The corresponding photon number in the cavity is given by

$$
N=2\left|A_{i n}\right|^{2} \frac{\kappa_{T}}{\kappa^{2}}
$$

\section{RESULTS AND DISCUSSION}

In the previous sections we have established the detection scheme and the trapping potential for an atom close to the microdisk (induced by surface and optical forces). In this case, the back-action of the atom on the light in the WGMs provides a means of atom detection.

There are several advantages of our detection scheme over a corresponding absorption detection. (i) It allows one to drive the atom far off-resonance, in which case the precise tuning of the disk resonator with respect to the atomic transition frequency is of minor importance. (ii) If the additional loss mechanisms are small compared to the disk-waveguide coupling strength [8], all of the pump light will leave the system through the forward waveguide output. Therefore a strong signal can be expected for most parameter regimes, which allows the use of standard photodetectors rather than sophisticated single-photon counters. (iii) The strong output signal also provides stability of the detection scheme against weak background scattering processes.

\subsection{Atom detection with only a blue-detuned mode}

To detect single atoms without trapping we can create an atom cloud in a magnetooptical trap above a photonic chip with a microdisk resonator and then drop this cloud. A certain number of atoms will then pass through the evanescent field of the microdisk resonator. First experiments of this type were described in Ref. 33 for Cs atoms.

The signal we are interested in is given by the difference of the detected photon numbers in the two arms of a heterodyne detector during the observation time $\tau$. The phase of the local oscillator is adjusted such that this difference is zero when no atom is interacting with the disk field. The presence of an atom is then inferred from a change in this intensity difference.

For a pump intensity less than $\sim 1 \mathrm{nW}$ the blue-detuned light forces are much smaller than the van der Waals (Casimir-Polder) forces over the relevant region where the atom interacts with the evanescent field. For this low intensity an atom is simply attracted to 

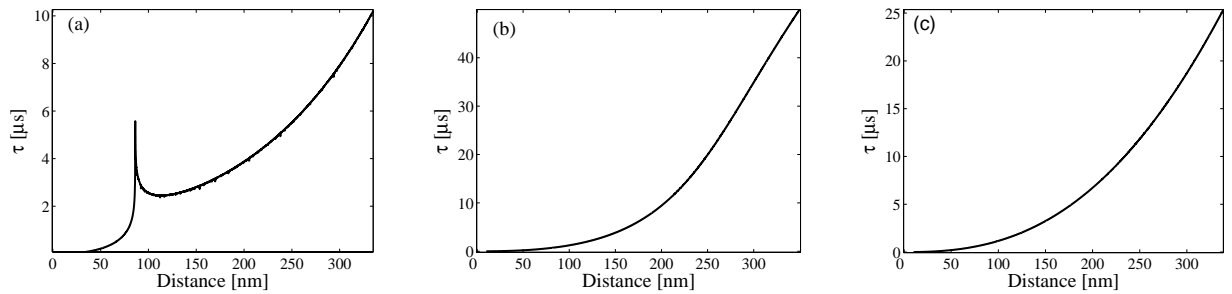

Fig. 4. Typical times for an atom to stay in the range of the evanescent field of the microdisk (0-500 $\mathrm{nm}$ from the disk edge) under the influence of surface and blue-detuned light potentials as in Fig. $2(x$ axis presents point of motion start, initial velocity in the direction towards the edge of the disk assumed to be zero). Pump intensities are a) $20 \mathrm{nW}$, b) $2 \mathrm{nW}$, c) $200 \mathrm{pW}$. The peak in

(a) is due to the extremum in the sum potential of Figs. 2(a) and 2(b).

the disk edge. For higher intensities, an atom initially placed at a distance further than $100-200 \mathrm{~nm}$ (Fig.2b), is pushed out of the system because of blue-detuned repulsion.

The typical time for an atom to stay within the range of the evanescent field of the microdisk under the influence of the surface and blue-detuned light potentials is presented in Fig. 4. The initial atom velocity in the direction towards the edge of the disk is assumed to be roughly zero. Obviously, taking the nonzero velocities into account will decrease this time for some of the atoms. There is an understandable correlation between the change in the gradient of the sum potentials presented in Fig. 2 (dashed line) and the time the atom stays within the range of the evanescent field of the blue-detuned light (taken to be 0-500nm from the disk edge). For a low intensity of $200 \mathrm{pW}$ (Fig. 2c), an atom will be attracted to the disk edge in a time interval of up to $25 \mu$ s depending on the initial atom position. As we increase the pump intensity of the blue-detuned light to $2 \mathrm{nW}$, the gradient of the sum potential decreases (Fig. 2d) and the time interval is increased up to $50 \mu \mathrm{s}$ ((Fig. 4b). For a strong pump intensity, a potential barrier is created with significant height relative to the atom energy (for cloud of ultra cold atoms, the temperature is typically $10-50 \mu \mathrm{K}$ ). In this case the atoms initially situated closer than $90 \mathrm{~nm}$ will be attracted to the disk edge in a time up to $6 \mu \mathrm{s}$. The atoms which are further than $90 \mathrm{~nm}$ (Fig. 2b) will be pushed from the system in a time interval of $3-10 \mu \mathrm{s}$. Our calculations show that the sum potential of the optical and surface potentials is strong enough to push the atoms situated closer than $\sim 60-120 \mathrm{~nm}$ to the disk surface in a time less than $2 \mu$ s depending on pump intensity.

The calculated coupling rate $g_{0} / 2 \pi$ at a distance of $\sim 70 \mathrm{~nm}$ is about $45 \mathrm{MHz}$. To compare experimental and theoretical values for $g_{0} / 2 \pi$ we have to take into account the change in the atom position during the measurement time.
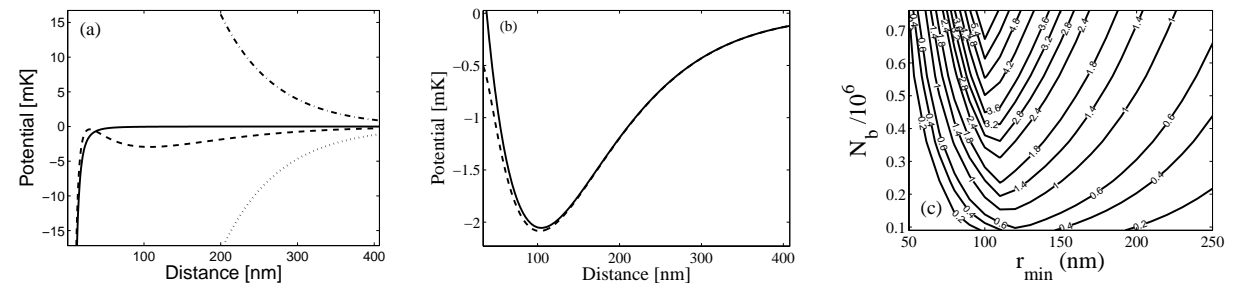

Fig. 5. Radial potentials near a 30- $\mu$ m disk: (a) Rb, potential formed by blue-detuned light (dash-dotted line) and red-detuned light (dotted), surface potential (solid), and sum potential (dashed). (b) Rb, the sum of the optical potentials solid), and the sum of the optical potentials and the surface potential (dashed). (c) Contour plot of the potential depth (in $\mathrm{mK}$ ) vs. position of the trap minimum $r_{\text {min }}$ and photon number $N_{b}$ for Rb atoms.

\subsection{Atom detection in a bi-chromatic optical trap}

Let us now assume that we hold an atom trapped in a two-mode optical potential at 
a given distance from the surface. In Fig. 5 we show sample potentials for a rubidium atom coupled to the $(l=167, q=1)$ blue-detuned mode and the $(l=163, q=1)$ red-detuned mode of a $30-\mu \mathrm{m}$ microdisk. The light intensities create a trapping potential of about 1.9 $\mathrm{mK}$ at a distance of $115 \mathrm{~nm}$ from the surface. The surface potential reduces the potential depth by lowering the potential barrier towards the disk surface and also slightly shifts the center position.

As an example, we present in Fig. 6 the detection of a single Rb atom coupled to a $30 \mu \mathrm{m}$ disk using the $(l=167, q=1)$ mode as the blue detuned detection light and the $(l=163, q=1)$ mode as the red detuned attractive trapping light. The gap between waveguide and disk is set to $0.5 \mu \mathrm{m}$, which gives sufficiently strong coupling to neglect surface back-scattering effects. The optical properties of these modes are given in Table 1. The pump intensity to create the trap is $\sim 2 \mu \mathrm{W}$.
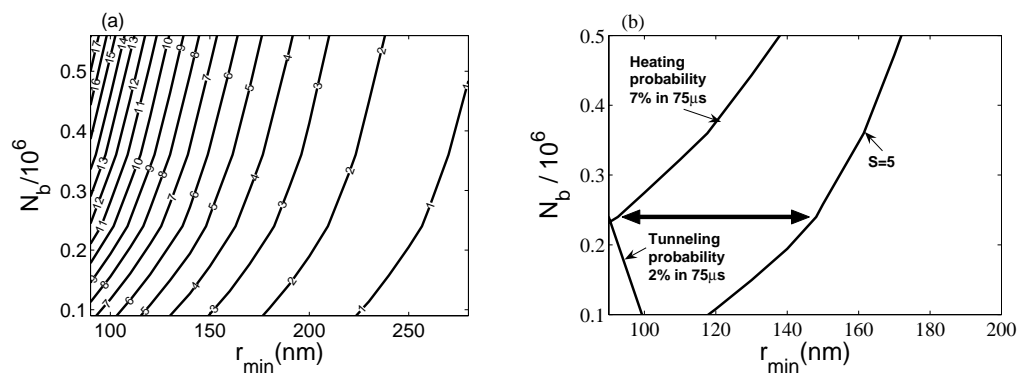

Figure 6: (a) Signal-to-noise ratio $S$, (b) performance characteristics. Both graphs display the data as a function of distance from the disk and blue-detuned photon number $N_{b}$ for Rb atoms. The integration time is $75 \mu \mathrm{s}$.

Figure 6(a) shows a contour plot of the signal-to-noise ratio for atom detection as defined by Eq. (15). Larger distances from the disk surface lead to weaker coupling because of the exponential decay of the evanescent fields and thus smaller values of $S$. Simultaneously, of course, the total number of spontaneously scattered photons $M$, Eq. (18), decreases with distance. However, the dependence of $S$ and $M$ on the system parameters are different, which allows us to optimize the atom-detection scheme.

The optimum parameter regime can be found from Fig. 6(b) where we combine the contour lines for $S=5$, heating probability $P_{\text {heat }}=7 \%$, and a tunnelling probability of $2 \%$ (see the Sec. 5.3 for details of this stability analysis). The area between the three curves, marked by the arrow in the figure, corresponds to system parameters where efficient singleatom detection is possible with very limited atom losses due to heating and tunnelling. The optimum point in the center of this area is found at blue-detuned photon number $N_{b}=2.4 \times 10^{5}$, red- detuned photon number $N_{r}=3.6 \times 10^{5}$, and a trap distance $r=120$ nm from the disk. For these parameters we obtain $S=7, P_{\text {heat }}=5.7 \%$, a potential depth of $1.6 \mathrm{mK}$, and harmonic oscillator frequencies $\left(\omega_{\mathrm{x}}, \omega_{\mathrm{y}}, \omega_{\mathrm{z}}\right)=2 \pi \times(0.92,4.2,0.11) \mathrm{MHz}$. We also calculated that the trap is stable under light intensity fluctuations up to $2 \%$.

\subsection{Trap stability}

There are several mechanisms by which a trapped atom can be lost from the trap. The most important of these are now discussed.

\subsubsection{Light-intensity balance}

As already discussed in the Sec. 5.2, the optical trapping potential relies on a delicate balance of two strong light fields which largely cancel each other. Using the exponential approximation of Eqs. (5) and (6), we find that a small fluctuation in one of the trapping fields, e.g., of the red-detuned field, will lead to a fluctuation of the trap center of

$$
\delta r_{\min }=\frac{1}{\alpha_{b}-\alpha_{r}} \frac{\delta V_{r 0}}{\left|V_{r 0}\right|}
$$


Because of the small difference between the two spatial decay coefficients $\alpha_{b}$ and $\alpha_{r}$, a small change of the magnitude of the potentials will significantly shift the minimum of the combined potential.

On the other hand, this sensitivity of the trap to the relative strengths of the blue and red detuned light fields allows us to accurately position an atom at a certain distance from the disk surface. For example, varying the intensity of the red-detuned light while keeping the intensity of the blue-detuned light constant will simultaneously change the trap depth as well as the trap position. An example of this ability to design the total trap potential is depicted in Fig. 5.

\subsubsection{Back-reflection from surface defects}

The delicate balance between the attractive and the repulsive optical potential required to form a shallow atom trap makes this type of trap highly susceptible to small fluctuations of the light intensities. Therefore, back-scattering of light at small surface defects can pose serious problems. A recent publication [41] has highlighted that back-scattered light with an intensity as low as 0.001 of the forward propagating mode intensity may decrease the potential depth by one half and consequently destroy the trap.

The back-scattering of light in a two-mode model (forward and backward propagating) has been discussed in Ref. 9. There it was estimated that for the latest generation of highQ micro-cavities [14-16], backscattering can be as low as $10^{-4}$, i.e., an order of magnitude below the limiting value derived in Ref. 41.

\subsubsection{Atom heating and tunneling}

Atoms can also be lost from the trap as a result of heating by the interaction with the light fields. Every photon of wave number $k$ emitted spontaneously by the atom will on average add one photon recoil energy $E_{r}=\hbar^{2} k^{2} /(2 m)$ to the kinetic energy of the photon. The total number of such events during an observation time $\tau$ is given by

$$
M=2 \Gamma \tau \frac{|\Omega|^{2}}{4 \Delta^{2}},
$$

where $2 \Gamma$ is the decay rate of the atomic excited state. A trap thus has to fulfill the condition $V>M E_{r}$ in order to hold an initially ultra-cold atom in the trapping potential during the interaction with the light fields.

In tightly confined traps, however, atoms are more likely to return to their initial motional state after a spontaneous emission event because of the Lamb-Dicke effect. If we assume a three-dimensional harmonic trapping potential, the probability that an atom in the harmonic ground state $\left|\psi_{0}\right\rangle$ returns into the same state after the emission of a photon with wave vector $\mathbf{k}$ is given by

$$
P_{0}(\mathbf{k})=\left|\left\langle\psi_{0}\left|e^{i \mathbf{k x}}\right| \psi_{0}\right\rangle\right|^{2}
$$

Therefore, the probability of heating the atom into a higher motional state after $M$ spontaneous emissions is

$$
P_{\text {heat }}(\tau)=1-P_{0}^{M} \text {. }
$$

For $P_{\text {heat }} \ll 1$, the atom will stay in the ground state during the detection process with high probability.

Another mechanism that can be significant for trapping stability is tunneling through the potential barrier to the disk surface. However, we find that this tunneling probability is negligible for typical values of the barrier height of $1-2 \mathrm{mK}$ and of the barrier width of $30-60 \mathrm{~nm}$.

\section{EXPERIMENTAL FEASIBILITY AND CONCLUSIONS}

We have shown that a single atom can be detected near the surface of a microdisk resonator with and without trapping, such that it can be done with negligible heating. 
The detection of the atom is done by a blue-detuned WGM of the resonator, while trapping at a fixed position is achieved by a second, red-detuned WGM. The two light fields create a trapping potential at a distance of $100-150 \mathrm{~nm}$ from the disk surface. At this distance, the atom-surface attractive interaction (van der Waals force) is much weaker than the light force, while the optical potential is sufficiently strong to create a deep trap for the atom. The atom is then confined in the radial direction and in the $z$ direction (perpendicular to the chip surface). For trapping in the tangential direction, we suggest that the red-light WGM would be coupled to the microdisk from both sides, such that a red-detuned standing wave is formed along the disk perimeter and the atom may be trapped in any of the maxima of the red detuned light.

The use of photonics for atom chips has been discussed recently [42]. Detection of atoms by evanescent fields has been achieved experimentally [43]. Moreover, the use of bi-chromatic light for guiding or trapping atoms has also been discussed before [35-41]. The idea of utilizing a two-dimensional microsphere, i.e., a disk or ring with a favorable fabrication feasibility, was put forward by us in recent papers together with a realistic tolerance analysis combining all the above ideas $[8,9]$. We note that state-of-the-art fabrication has reached a point where previous concerns regarding the spatial instability of the trapping potential due to light back scatter from imperfections [41] may be no longer valid. Concerning the required light mode stability, we estimate an acceptable tolerance of $\sim 2 \%$. This has been calculated when demanding high stability for the trap parameters as well as atom-light interaction, and is highly dependent on surface roughness and mode coupling.

On the experimental side, recent work has already demonstrated strong coupling of single Cs atoms with monolithic microtoroidal resonators that have a capability of input-output coupling with small parasitic losses, and a demonstrated ideality of more than 99.97 [32, 33]. However, these experiments were performed at very small detuning and pump intensity. Atoms were detected by high-sensitivity photon counters with signals as low as 6 photons.

Our work suggests that experiments at largely increased detuning of optical modes from atomic transitions will allow for single-atom detection with higher pump intensity and thus with far less sophisticated detectors. On the other hand, if input field intensities become too high, unwanted nonlinear effects start to play a role, such as instabilities from Kerr optical parametric oscillation [44] and radiation pressure induced mechanical oscillation [45].

Further work will also need to address in detail the issue of loading, i.e, how the atoms are brought close to the disk surface. This loading is crucial as it deals with the interplay between the specific atom optics elements such as guides and traps, and the detector responsible for extracting the signal.

\section{References}

[1] R. E. Slusher, A. F. J. Levi, U. Mohideen, S. L. McCall, S. J. Pearton, and R. A. Logan, "Tunable optical filters for dense WDM networks," Appl. Phys. Lett. 63, 1310-1312 (1993) [doi:10.1063/1.109714].

[2] D. Sadot and E. Boimovich, "Threshold characteristics of semiconductor microdisk lasers," IEEE Comm. Magazine 36, 50-55 (1998).

[3] F. Vollmer, D. Braun, A. Libchaber, M. Khoshsima, I. Teraoka, and S. Arnold, "Protein detection by optical shift of a resonant microcavity," Appl. Phys. Lett. 80, 4057-4059 (2002) [doi:10.1063/1.1482797].

[4] R. Folman, P. Krüger, J. Schmiedmayer, J. Denschlag, and C. Henkel, "Microscopic atom optics: From Wires to an atom chip," Adv. At. Mol. Opt. Phys. 48, 263-356 (2002).

[5] J. Reichel, "Microchip traps and Bose-Einstein condensation," Appl. Phys. B 74, 469-487 (2002) [doi:10.1007/s003400200861].

[6] P. Horak, B. G. Klappauf, A. Haase, R. Folman, J. Schmiedmayer, P. Domokos, and E. A. Hinds, "Possibility of single-atom detection on a chip," Phys. Rev. A 67, 043806 (2003) [doi:10.1103/PhysRevA.67.043806].

[7] B. Lev, K. Srinivasan, P. Barclay, O. Painter, and H. Mabuchi, "Feasibility of detecting single atoms using photonic bandgap cavities," Nanotechnology 15, S556-S561 (2004) [doi:10.1088/0957-4484/15/10/010].

[8] M. Rosenblit, P. Horak, S. Helsby, and R. Folman, "Single-atom detection using whispering-gallery modes of microdisk resonators," Phys. Rev. A 70, 053808 (2004) [doi:10.1103/PhysRevA.70.053808]. 
[9] M. Rosenblit, Y. Japha, P. Horak, and R. Folman, "Simultaneous optical trapping and detection of atoms by microdisk resonators," Phys. Rev. A 73, 063805 (2006) [doi:10.1103/PhysRevA.73.063805].

[10] V. Lefevre-Seguin and S. Haroche, "Towards cavity-QED experiments with silica microspheres," Mater. Sci. Eng. B 48, 53-58 (1997) [doi:10.1016/S0921-5107(97)00080-9].

[11] W. von Klitzing, R. Long, V. S. Ilchenko, J. Hare, and V. Lefevre-Seguin, "Tunable whispering gallery modes for spectroscopy and CQED experiments," New J. Phys. 3, 14 (2001) [doi:10.1088/1367-2630/3/1/314].

[12] D. W. Vernooy, V. S. Ilchenko, H. Mabuchi, E. W. Streed, and H. J. Kimble, "High-Q measurements of fused-silica microspheres in the near infrared," Opt. Lett. 23, 247-249 (1998).

[13] D. W. Vernooy, A. Furusawa, N. P. Georgiades, V. S. Ilchenko, and H. J. Kimble, "Cavity QED with high-Q whispering gallery modes," Phys. Rev. A 57, R2293-R2296 (1998) [doi:10.1103/PhysRevA.57.R2293].

[14] T. J. Kippenberg, S. M. Spillane, D. K. Armani, and K. J. Vahala, "Fabrication and coupling to planar high-Q silica disk microcavities," Appl. Phys. Lett. 83, 797-799 (2003) [doi:10.1063/1.1593833].

[15] D.K. Armani, T.J. Kippenberg, S. M. Spillane, and K. J. Vahala, "Ultra-high-Q toroid microcavity on a chip," Nature 421, 925-928 (2003) [doi:10.1038/nature01371].

[16] V. Zwiller, S. Fälth, J. Persson, W. Seifert, L. Samuelson, and G. Björk, "Fabrication and time-resolved studies of visible microdisk lasers," J. Appl. Phys. 93, 2307-2309 (2003).

[17] T. Pellizzari, S. A. Gardiner, J. I. Cirac, and P. Zoller, "Decoherence, continuous observation, and quantum computing: A cavity QED model," Phys. Rev. Lett. 75 , 3788-3791 (1995) [doi:10.1103/PhysRevLett.75.3788].

[18] S. J. van Enk, J. I. Cirac, and P. Zoller, "Photonic channels for quantum communication," Science 279, 205-208 (1998) [doi:10.1126/science.279.5348.205].

[19] C. J. Hood, M. S. Chapman, T. W. Lynn, and H. J. Kimble, "Real-time cavity QED with single atoms," Phys. Rev. Lett. 80, 4157-4160 (1998) [doi:10.1103/PhysRevLett.80.4157].

[20] P. W. H. Pinkse, T. Fischer, P. Maunz, and G. Rempe, "Trapping an atom with single photons," Nature 404, 365-368 (2000) [doi:10.1038/35006006].

[21] M. Pelton, C. Santori, G. S. Solomon, O. Benson, and Y. Yamamoto, "Triggered single photons and entangled photons from a quantum dot microcavity," Eur. Phys. J. 18, 179-190 (2002) [doi:10.1140/epjd/e20020022].

[22] P. Michler, A. Kiraz, C. Becher, L. D. Zhang, E. Hu, A. Imamoglu, W. V. Schoenfeld, and P. M. Petroff, "Quantum dot lasers using high-Q microdisk cavities," Phys. Status Solidi B 224, 797-801 (2001) [doi:10.1002/(SICI)1521-3951(200104)224:3ز797::AID-PSSB797¡3.0.CO;2-I].

[23] J. McKeever, J. R. Buck, A. D. Boozer, and H. J. Kimble, "Determination of the number of atoms trapped in an optical cavity," Phys. Rev. Lett. 93, 143601 (2004) [doi:10.1103/PhysRevLett.93.143601].

[24] A. Peters, K. Y. Chung, and S. Chu, "Measurement of gravitational acceleration by dropping atoms," Nature 400, 849-852 (1999) [doi:10.1038/23655].

[25] T. L. Gustavson, A. Landragin, and M. A. Kasevich, "Rotation sensing with a dual atom-interferometer Sagnac gyroscope," Class. Quant. Grav. 17, 2385-2398 (2000) and references therein [doi:10.1088/0264-9381/17/12/311].

[26] E. A. Hinds, C. J. Vale, and M. G. Boshier, "Two-wire waveguide and interferometer for cold atoms," Phys. Rev. Lett. 86, 1462-1464 (2001) [doi:10.1103/PhysRevLett.86.1462].

[27] W. Hänsel, J. Reichel, P. Hommelhoff, and T. W. Hänsch, "Trapped-atom interferometer in a magnetic microtrap," Phys. Rev. A 64, 063607 (2001).

[28] E. Andersson, T. Calarco, R. Folman, M. Andersson, B. Hessmo, and J. Schmiedmayer, "Multimode interferometer for guided matter waves," Phys. Rev. Lett. 88, 100401 (2002) [doi:10.1103/PhysRevLett.88.100401]. 
[29] G. Santarelli, Ph. Laurent, P. Lemonde, A. Clairon, A. G. Mann, S. Chang, A. N. Luiten, and C. Salomon, "Quantum projection noise in an atomic fountain. A high stability cesium frequency standard," Phys. Rev. Lett. 82, 4619-4622 (1999) [doi:10.1103/PhysRevLett.82.4619].

[30] A. M. Steane, "Quantum computing," Rep. Prog. Phys. 61, 117-173 (1998) [doi:10.1088/0034-4885/61/2/002].

[31] M. B. Plenio and V. Vedral, "Teleportation, entanglement and thermodynamics in the quantum world," Contemp. Phys. 39, 431-446 (1998) [doi:10.1080/001075198181766].

[32] P. E. Barclay, K. Srinivasan, O. Painter, B. Lev, and H. Mabuchi, "Integration of fiber-coupled high-Q SiNx microdisks with atom chips," Appl. Phys. Lett. 89, 131108 (2006) [doi:10.1063/1.2356892].

[33] T. Aoki, B. Dayan, E. Wilcut, W. P. Bowen, A. S. Parkins, T. J. Kippenberg, K. J. Vahala and H. J. Kimble, "Observation of strong coupling between one atom and a monolithic microresonator," Nature 443, 671-674 (2006) [doi:10.1038/nature05147].

[34] S.-T. Wu and C. Eberlein, "Quantum electrodynamics of an atom in front of a non-dispersive dielectric half-space," Proc. R. Soc. Lond. A 455, 2487-2512 (1999) [doi: 10.1098/rspa.1999.0413].

[35] Y. B. Ovchinnikov, S. V. Shulga, and V. I. Balykin, "An atomic trap based on evanescent light waves," J. Phys. B 24, 3173-3178 (1991) [doi:10.1088/0953-4075/24/14/009].

[36] D. W. Vernooy and H. J. Kimble, "Quantum structure and dynamics for atom galleries," Phys. Rev. A 55, 1239-1261 (1997) [doi:10.1103/PhysRevA.55.1239].

[37] H. Mabuchi and H. J. Kimble, "Atom galleries for whispering atoms: binding atoms in stable orbits around an optical resonator," Opt. Lett. 19, 749-751 (1994).

[38] A. H. Barnett, S. P. Smith, M. Olshanii, K. S. Johnson, A. W. Adams, and M. Prentiss, "Substrate-based atom waveguide using guided two-color evanescent light fields," Phys. Rev. A 61, 023608 (2000) [doi:10.1103/PhysRevA.61.023608].

[39] F. Le Kien, V. I. Balykin, and K. Hakuta, "Atom trap and waveguide using a two-color evanescent light field around a subwavelength-diameter optical fiber," Phys. Rev. A 70, 063403 (2004) [doi:10.1103/PhysRevA.70.063403].

[40] P. Domokos and H. Ritsch, "Efficient loading and cooling in a dynamic optical evanescent-wave microtrap," Europhys. Lett. 54, 306-312 (2001) [doi:10.1209/epl/i2001-00242-8].

[41] J. P. Burke, Jr., S.-T. Chu, G. W. Bryant, C. J. Williams, and P. S. Julienne, "Designing neutral-atom nanotraps with integrated optical waveguides," Phys. Rev. A 65, 043411 (2002) [doi:10.1103/PhysRevA.65.043411].

[42] G. Birkl, F. B. G. Buchkremer, R. Dumke, and W. Ertmer, "Atom optics with microfabricated optical elements," Opt. Commun. 191, 67-81 (2001) [doi:10.1016/S0030-4018(01)01107-5].

[43] R. A. Cornelussen, A. H. van Amerongen, B. T. Wolschrijn, R. J. C. Spreeuw, and H. B. van Linden van den Heuvell, "Cold trapped atoms detected with evanescent waves," Eur. Phys. J. D 21, 347-351 (2002) [doi:10.1140/epjd/e2002-00205-1].

[44] T. J. Kippenberg, S. M. Spillane, and K. J. Vahala, "Kerr-nonlinearity optical parametric oscillation in an ultrahigh-Q toroid microcavity," Phys. Rev. Lett. 93, 083904 (2004) [doi:10.1103/PhysRevLett.93.083904].

[45] T. J. Kippenberg, H. Rokhsari, T. Carmon, A. Scherer, and K. J. Vahala, "Analysis of radiation-pressure induced mechanical oscillation of an optical microcavity," Phys. Rev. Lett. 95, 033901 (2005) [doi:10.1103/PhysRevLett.95.033901].

Michael Rosenblit is a Researcher at the Ilse Katz Center for Meso- and Nanoscale Science and Technology, Ben-Gurion University of the Negev, Israel. He received his Ph.D. degree in chemical physics from the Tel-Aviv University in 1996. He is the author of more than 20 journal papers and book chapters, and 6 patents. His current research interests include atom chip photonics, quantum optics, ultracold finite systems, micro resonant cavities, tunable nano devices. 
Peter Horak is a Senior Research Fellow at the Optoelectronics Research Centre, University of Southampton, UK. He received his Ph.D. degree in theoretical quantum optics from the University of Innsbruck, Austria, in 1998. His main research interest is in theory and modelling of nonlinear and quantum optical phenomena and devices. His current research includes work on micro-resonators and their applications for sensing and quantum information processing, holey fibers, nonlinear fiber optics, short-pulse propagation, and noise properties of optoelectronics devices. He is the author of over 100 journal and conference papers.

Eyal Fleminger is a student currently concluding his M.Sc. degree in the Atom Chip Group of the Department of Physics, Ben-Gurion University of the Negev, Israel. He received his B.Sc. degree in physics at Ben-Gurion University in 2003. His current research includes design/modelling and experimental work on magnetic and magneto-optical traps for ultracold atoms, as well as atom chip photonics.

Yonathan Japha is a Researcher at the Department of Physics, Ben-Gurion University of the Negev, Israel. He received his Ph.D. degree in chemical physics from the the Weizmann institute of Science, Rehovot in 1997. He is the author of more than 20 journal and conference papers. His research interests are in the theory of ultracold atoms in magnetic and electromagnetic fields, the theory of noise and decoherence of cold atoms, and applications of theory and simulation to the design and optimization of structures on an atom chip, e.g., atom interferometry.

Ron Folman is a Researcher at the Department of Physics, Ben-Gurion University of the Negev, Israel. He received his Ph.D. degree in physics from the the Weizmann institute of Science, Rehovot in 1998, after which he did his post-doc in the university of Innsbruck following which he was a researcher for 3 years at the university of Heidelberg. He is the author of more than 40 journal and conference papers. His research interests are in theory and experiment into the foundations of quantum mechanics. His main experimental expertise is in ultracold atoms, photonics and nano-fabrication. 